\title{
On the orbits of an orthogonal group action
}

\author{
Kyle Czarnecki, R. Michael Howe and Aaron McTavish
}

(Communicated by Józef H. Przytycki)

Let $G$ be the Lie group $\mathrm{SO}(n, \mathbb{R}) \times \mathrm{SO}(n, \mathbb{R})$ and let $V$ be the vector space of $n \times n$ real matrices. An action of $G$ on $V$ is given by

$$
(g, h) \cdot v:=g^{-1} v h, \quad(g, h) \in G, \quad v \in V .
$$

We consider the orbits of this group action and demonstrate a cross-section to the orbits. We then determine the stabilizer for a typical element in this crosssection and completely describe the fundamental group of an orbit of maximal dimension.

\section{Introduction}

Let $G$ be the Lie group $\mathrm{SO}(n, \mathbb{R}) \times \mathrm{SO}(n, \mathbb{R})$ and let $V$ be the vector space of $n \times n$ real matrices. An action of $G$ on $V$ is given by

$$
(g, h) . v:=g^{t} v h=g^{-1} v h, \quad(g, h) \in G, \quad v \in V,
$$

where $g^{t}$ denotes the matrix transpose of $g$ and where the operation on the right is matrix multiplication. This action is obviously smooth (having continuous derivatives of all orders) since the matrix entries in $(g, h) . v$ are polynomial functions of the matrix entries of $g, h$ and $v$.

For each $v \in V$ we define the orbit of $v$, denoted by $G . v \subseteq V$, as the set

$$
G . v:=\{(g, h) . v \mid(g, h) \in G\} .
$$

For $v, w \in V$ the relation

$$
v \sim w \text { if } v \text { and } w \text { are in the same } G \text {-orbit }
$$

MSC2000: primary 22C05, 57S15; secondary 55Q52.

Keywords: representation theory, orbit, Lie group, homotopy group, Clifford algebra.

This research was conducted in part during the 2007 summer Undergraduate Research Experience in Pure and Applied Mathematics at the University of Wisconsin - Eau Claire, supported by NSF-REU grant DMS-0552350 and the Office of Research and Sponsored Programs (UW - Eau Claire). 
is an equivalence relation and so $V$ is partitioned into $G$-orbits. We also define $G_{v}$, the stabilizer of $v$, to be the those elements in $G$ that fix $v$ :

$$
G_{v}=:\{(g, h) \in G \mid(g, h) . v=v\} .
$$

For each $v \in V, G_{v}$ is a closed (usually not normal) subgroup of $G$, and so is a Lie group.

Let $G / G_{v}$ denote the set of left cosets of $G_{v}$ in $G$. Since $G_{v}$ is a closed subgroup of $G, G / G_{v}$ is a differentiable manifold and $\operatorname{dim} G / G_{v}=\operatorname{dim} G-\operatorname{dim} G_{v}$, where dim indicates the dimension. Furthermore, $G / G_{v}$ is diffeomorphic to the orbit $G . v$. If $G_{v}$ is normal in $G$, then $G / G_{v}$ is a Lie group [Bröcker and tom Dieck 1985, Section1.4].

A subset $D$ of $V$ is a cross-section to the orbits if every $G$-orbit intersects $D$. That is, for each $v \in V$ there is an element $(g, h) \in G$ and an element $d \in D$ such that $(g, h) . v=d$. Some definitions of a cross-section are more restrictive, requiring that each orbit intersect the cross-section exactly once.

In this paper we consider the orbits of this group action. In Section 2 we demonstrate a cross-section of the orbits, and in Section 3 we determine the stabilizer for a typical element in this cross-section. In Section 4 we discuss the orbits for the case $n=2$ and introduce generic orbits - those of maximal dimension - for arbitrary $n$. Section 5 reviews some useful information about fundamental groups, covering spaces, and the covering group $\operatorname{Spin}(n)$. Our main result is in Section 6 where we connect these ideas in order to completely describe the fundamental group of a generic orbit, and in Section 7 we work through an example that further exposes the anatomy. We close with a few remarks in Section 8 regarding those orbits that do not have maximal dimension.

\section{Cross section to the orbits}

In this section we show that the diagonal matrices with non-negative entries constitute a cross-section to the group action.

Proposition 2.1. Let $G=\mathrm{SO}(n) \times \mathrm{SO}(n)$ and let $V$ be the vector space of $n \times n$ real matrices. Let $G$ act on $V$ via $(g, h) . v=g^{t} v h$. Then for each $v \in V$ there is a $\left(k_{1}, k\right) \in G$ such that $\left(k_{1}, k\right) . v=\operatorname{diagonal}\left(d_{1}, \ldots, d_{n}\right)$, with $d_{1} \geq d_{2} \geq \cdots \geq d_{n} \geq 0$.

Proof. Let $v \in G L(n, \mathbb{R})$ where $G L(n, \mathbb{R})$ is the (dense, open) subset of invertible $n \times n$ matrices in $V$. Then $v^{t} v$ is a symmetric matrix with positive eigenvalues, and hence is diagonalizable via conjugation by an element in $\operatorname{SO}(n, \mathbb{R})$. That is, there is a $k$ in $\operatorname{SO}(n, \mathbb{R})$ such that

$$
k^{t} v^{t} v k=a,
$$

where $a=\operatorname{diagonal}\left(a_{1}, \ldots, a_{n}\right)$ with $a_{1} \geq a_{2} \geq \cdots \geq a_{n}>0$. 
Now let $a^{-1 / 2}=\operatorname{diagonal}\left(1 / \sqrt{a_{1}}, \ldots, 1 / \sqrt{a_{n}}\right)$. If $\Phi_{n}$ is the $n \times n$ identity matrix we have

$$
\Phi_{n}=a^{-1 / 2} a a^{-1 / 2}=a^{-1 / 2}\left[k^{t} v^{t} v k\right] a^{-1 / 2}=\left(v k a^{-1 / 2}\right)^{t} v k a^{-1 / 2} .
$$

It follows that $v k a^{-1 / 2}$ is in $O(n, \mathbb{R})$. Let $a^{1 / 2}=\operatorname{diagonal}\left(\sqrt{a_{1}}, \ldots, \sqrt{a_{n}}\right)$. Then

$$
a^{1 / 2}=\mathscr{I}_{n} a^{1 / 2}=\left[a^{-1 / 2} k^{t} v^{t} v k a^{-1 / 2}\right] a^{1 / 2}=a^{-1 / 2} k^{t} v^{t} v k .
$$

Thus, if $k_{1}=v k a^{-1 / 2}$, we can write this as

$$
\left(k_{1}\right)^{t} v k=\left(k_{1}, k\right) \cdot v=a^{1 / 2},
$$

where $k_{1} \in O(n, \mathbb{R})$ and $k \in \mathrm{SO}(n, \mathbb{R})$. If $k_{1}$ happens to be in $\mathrm{SO}(n, \mathbb{R})$ we are done. If not, we can change the sign of one of the entries in $a^{-1 / 2}$ so that $k_{1}$ is in $\mathrm{SO}(n, \mathbb{R})$, proving the result for any $V$ in the dense subset of invertible $n \times n$ matrices. Since our group action is continuous, the result holds for all $v \in V$. We could also modify the above proof slightly to account for those eigenvalues of $v^{t} v$ that are equal to zero.

\section{The stabilizer of a representative element}

Let $\Gamma$ be an arbitrary group acting on a set $X$. If $x$ and $y$ are in the same $\Gamma$-orbit, then $x=\gamma . y$ for some $\gamma \in \Gamma$. It is a standard result that $\gamma^{-1} \Gamma_{x} \gamma=\Gamma_{y}$, that is, the stabilizers are isomorphic via conjugation. Therefore, it is sufficient to determine the stabilizers of those elements that are in the cross section.

We start with a simple example that demonstrates the general idea for the situation that we are considering. Let $d \in V$ and $(g, h) \in G$ be given by

$$
\begin{gathered}
d=\left(\begin{array}{ccc}
d_{1} & 0 & 0 \\
0 & d_{1} & 0 \\
0 & 0 & d_{2}
\end{array}\right), \quad \text { where } d_{1}>d_{2}>0, \\
g=\left(\begin{array}{lll}
g_{1,1} & g_{1,2} & g_{1,3} \\
g_{2,1} & g_{2,2} & g_{2,3} \\
g_{3,1} & g_{3,2} & g_{3,3}
\end{array}\right), \quad h=\left(\begin{array}{lll}
h_{1,1} & h_{1,2} & h_{1,3} \\
h_{2,1} & h_{2,2} & h_{2,3} \\
h_{3,1} & h_{3,2} & h_{3,3}
\end{array}\right) .
\end{gathered}
$$

We may assume $d_{1}>d_{2}$ since conjugation by a matrix such as

$$
\left(\begin{array}{rrr}
0 & 0 & -1 \\
0 & 1 & 0 \\
1 & 0 & 0
\end{array}\right) \in \operatorname{SO}(3)
$$

will reorder the entries in $d$.

If $(g, h)$ stabilizes $d$ then $g^{t} d h=d$ or equivalently, $d h=g d$, so we have 


$$
\left(\begin{array}{lll}
d_{1} h_{1,1} & d_{1} h_{1,2} & d_{1} h_{1,3} \\
d_{1} h_{2,1} & d_{1} h_{2,2} & d_{1} h_{2,3} \\
d_{2} h_{3,1} & d_{2} h_{3,2} & d_{2} h_{3,3}
\end{array}\right)=\left(\begin{array}{lll}
d_{1} g_{1,1} & d_{1} g_{1,2} & d_{2} g_{1,3} \\
d_{1} g_{2,1} & d_{1} g_{2,2} & d_{2} g_{2,3} \\
d_{1} g_{3,1} & d_{1} g_{3,2} & d_{2} g_{3,3}
\end{array}\right) .
$$

That is, the first entry in $d$ acts on the first row of $h$, but acts on the first column of $g$, etc. The rows of $g$ and $h$ are orthonormal (considered as vectors in $\mathbb{R}^{3}$ with the usual dot product), and we compare the squared length of the first row of $d h$ with the first row of $g d$ in (3-1):

$$
\left(d_{1} h_{1,1}\right)^{2}+\left(d_{1} h_{1,2}\right)^{2}+\left(d_{1} h_{1,3}\right)^{2}=\left(d_{1} g_{1,1}\right)^{2}+\left(d_{1} g_{1,2}\right)^{2}+\left(d_{2} g_{1,3}\right)^{2} .
$$

Since first rows of both $h$ and $g$ have length 1, we have

$$
\begin{aligned}
\Rightarrow\left(d_{1}\right)^{2} & =\left(d_{1}\right)^{2}\left[\left(h_{1,1}\right)^{2}+\left(h_{1,2}\right)^{2}+\left(h_{1,3}\right)^{2}\right] \\
& =\left(d_{1} g_{1,1}\right)^{2}+\left(d_{1} g_{1,2}\right)^{2}+\left(d_{2} g_{1,3}\right)^{2}<\left(d_{1}\right)^{2},
\end{aligned}
$$

since $d_{1}>d_{2}$. But this is impossible unless $g_{1,3}=0$, and hence $h_{1,3}=0$. Comparing the lengths of the second rows shows that $g_{2,3}=h_{2,3}=0$, and applying this same reasoning to the columns gives $h_{3,1}=g_{3,1}=0$ and $h_{3,2}=g_{3,2}=0$.

We now have

$$
\left(\begin{array}{ccc}
d_{1} h_{1,1} & d_{1} h_{1,2} & 0 \\
d_{1} h_{2,1} & d_{1} h_{2,2} & 0 \\
0 & 0 & d_{2} h_{3,3}
\end{array}\right)=\left(\begin{array}{ccc}
d_{1} g_{1,1} & d_{1} g_{1,2} & 0 \\
d_{1} g_{2,1} & d_{1} g_{2,2} & 0 \\
0 & 0 & d_{2} g_{3,3}
\end{array}\right),
$$

which immediately implies that $h=g$. The condition that $g^{t} g=I$ gives us that each of the block submatrices must be orthogonal, and of course $g$ must have determinant 1 . Note that if we were to allow $d_{2}=0$ then $g_{3,3}$ and $h_{3,3}$ need not be equal.

An inductive argument on the different eigenvalues of $d$ proves the general case and is not particularly enlightening, so we state the following result.

Proposition 3.1. Let $G=\mathrm{SO}(n) \times \mathrm{SO}(n)$ and let $V$ be the vector space of $n \times n$ real matrices. Let $G$ act on V via $(g, h) . v=g^{t} v h$. Let

$$
d=\operatorname{diagonal}(\underbrace{d_{1}, \ldots, d_{1}}_{s_{1}}, \ldots, \underbrace{d_{k}, \ldots, d_{k}}_{s_{k}}) \in V
$$

with $d_{1}>d_{2}>\ldots>d_{k} \geq 0$, and let $G_{d}$ be the stabilizer of $d$ in $G$. If $d_{k}>0$, then $G_{d}=\left\{(g, g): g \in S\left(O\left(s_{1}\right) \times \cdots \times O\left(s_{k}\right)\right)\right\}$.

That is, each $g$ consists of block-diagonal matrices where each block is an $s_{i} \times s_{i}$ orthogonal matrix and where $s_{i}$ is the multiplicity of the eigenvalue $d_{i}$ in $d$. The " $S$ " indicates that the product of the determinants of the blocks is 1 . If $d_{k}=0$ then $G_{d}=(g, h)$ where $g$ and $h$ consist of block-diagonal matrices with each $i$-th block in $O\left(s_{i}\right)$, and where $g=h$ except for the $k$-th block. 


\section{Orbits}

A natural question is "What are these orbits like?" From the introduction we know that, for any element $v \in V$, the orbit $G . v$ is diffeomorphic to the coset space $G / G_{v}$, with $\operatorname{dim} G . v=\operatorname{dim} G-\operatorname{dim} G_{v}$. Since any two elements in the same $G$ orbit have isomorphic stabilizers, it will be sufficient to consider the orbits of those representative elements $d$ in the cross-section $D$. In particular, the dimension of these orbits is completely determined by the multiplicity of the distinct eigenvalues of $d$ and is independent of their actual values.

Example: $\boldsymbol{n}=2$. In low-dimensional cases we can use computer graphics to get an idea about the nature of these orbits, and we now illustrate this for the twodimensional Lie group $G=\mathrm{SO}(2) \times \mathrm{SO}(2)$. Figure 1 shows the orbit of $d=\left(\begin{array}{ll}1 & 0 \\ 0 & 0\end{array}\right)$, with a cut-away view on the right. Note that, for $n=2$, the orbit lies in $\operatorname{Mat}(2, \mathbb{R}) \cong$ $\mathbb{R}^{4}$, and each figure is a projection of this orbit onto $\mathbb{R}^{2}$. Since $\mathrm{G}$ is abelian, $G_{d}$ is normal in $G$ and so $G / G_{d}$ is an abelian Lie group which is compact since the quotient map is continuous. Since $G_{d}=\left\{\left(\Phi_{2}, \Phi_{2}\right),\left(-\Phi_{2},-\Phi_{2}\right)\right\}$ which is discrete, the orbit $G . d$ has dimension 2. We conclude that this orbit is diffeomorphic to the 2-torus embedded in $\mathbb{R}^{4}$, since this is the only two-dimensional compact abelian Lie group. Notice that the graphics could be misleading, since we usually picture the 2-torus in $\mathbb{R}^{3}$ as resembling the surface of a donut.

Note that if an element $d$ in the cross-section $D$ has only one eigenvalue, then the stabilizer $G_{d}$ is isomorphic to $\mathrm{SO}(2)$ and so the orbit $G . d$ is one-dimensional and is diffeomorphic to $\mathrm{SO}(2)$, that is, a circle.

Generic orbits. We now move on to consider the following special case of generic orbits - those with maximal dimension - for arbitrary $n$. We will reserve the symbol $\delta$ for a diagonal matrix in the cross-section $D$ with $n$ distinct eigenvalues.
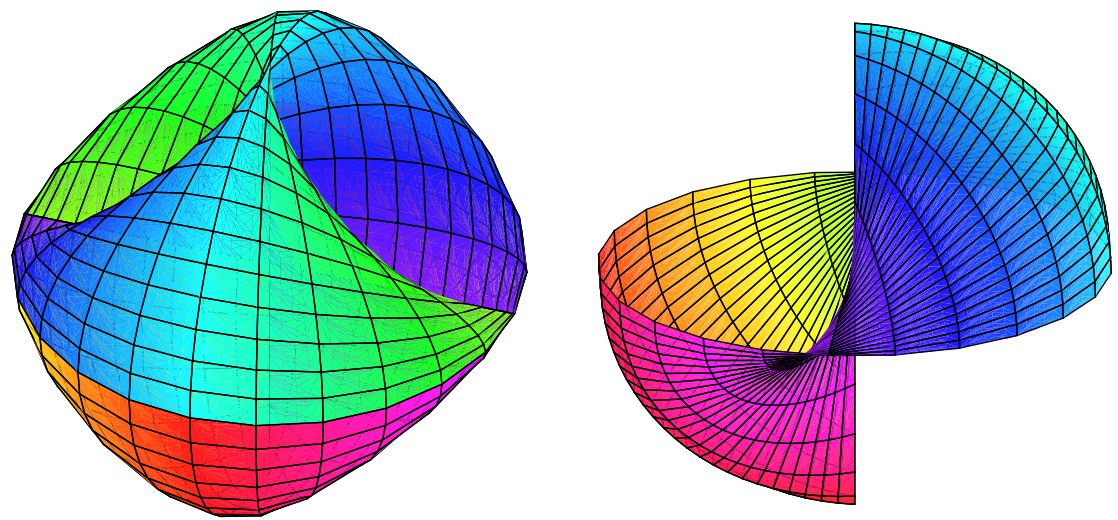

Figure 1. An orbit for $n=2$ projected onto $\mathbb{R}^{2}$. Right: cut-away view of same orbit. 
That is, $\delta=\operatorname{diagonal}\left(d_{1}, \ldots, d_{n}\right)$ with $d_{1}>d_{2}>\cdots>d_{n} \geq 0$. From Proposition 3.1 we have $G_{\delta}=(g, g)$, where $g=\operatorname{diagonal}( \pm 1, \ldots, \pm 1)$ has an even number of entries equal to -1 . Since the stabilizer of $\delta$ is discrete, the dimension of the $G$-orbit of $\delta$ is equal to the dimension of $G$.

Proposition 4.1. Let $G=\mathrm{SO}(n) \times \mathrm{SO}(n)$ and let $V$ be the vector space of $n \times n$ real matrices. Let $G$ act on $V$ via $(g, h) . v=g^{t} v h$. Let

$$
\delta=\operatorname{diagonal}\left(d_{1}, d_{2}, \ldots, d_{n}\right) \in V
$$

with $d_{1}>d_{2}>\cdots>d_{n} \geq 0$, and let $G_{\delta}$ be the stabilizer of $\delta$ in $G$. Then $\left|G_{\delta}\right|$, the order of $G_{\delta}$, is $2^{n-1}$.

Proof. From Proposition 3.1, $G_{\delta}$ consists of $n$ copies of $O(1)= \pm 1$ lying in $\mathrm{SO}(n)$, so there must be an even number of entries equal to -1 . Thus

$$
\left|G_{\delta}\right|=\left(\begin{array}{l}
n \\
0
\end{array}\right)+\left(\begin{array}{l}
n \\
2
\end{array}\right)+\left(\begin{array}{l}
n \\
4
\end{array}\right)+\cdots+\left(\begin{array}{l}
n \\
k
\end{array}\right)
$$

where $k=n$ if $n$ is even and $k=n-1$ if $n$ is odd. From the binomial theorem,

$$
\begin{aligned}
2^{n} & =(1+1)^{n}+(1-1)^{n} \\
& =\left[\left(\begin{array}{l}
n \\
0
\end{array}\right)+\left(\begin{array}{c}
n \\
1
\end{array}\right)+\left(\begin{array}{c}
n \\
2
\end{array}\right)+\cdots+\left(\begin{array}{l}
n \\
n
\end{array}\right)\right]+\left[\left(\begin{array}{c}
n \\
0
\end{array}\right)-\left(\begin{array}{c}
n \\
1
\end{array}\right)+\left(\begin{array}{c}
n \\
2
\end{array}\right)-\cdots \pm\left(\begin{array}{l}
n \\
n
\end{array}\right)\right] \\
& =2\left[\left(\begin{array}{l}
n \\
0
\end{array}\right)+\left(\begin{array}{c}
n \\
2
\end{array}\right)+\left(\begin{array}{c}
n \\
4
\end{array}\right)+\cdots+\left(\begin{array}{l}
n \\
k
\end{array}\right)\right]=2\left|G_{\delta}\right| .
\end{aligned}
$$

Again, what are these orbits like? Figure 2 shows a (projection of a) two-dimensional slice of the orbit of $\delta=\operatorname{diagonal}(2,1,0)$ for the case $n=3$. Could this be just a torus in disguise, as was the case $n=2$ ? One way to determine how interesting the orbits are is to consider their fundamental groups.
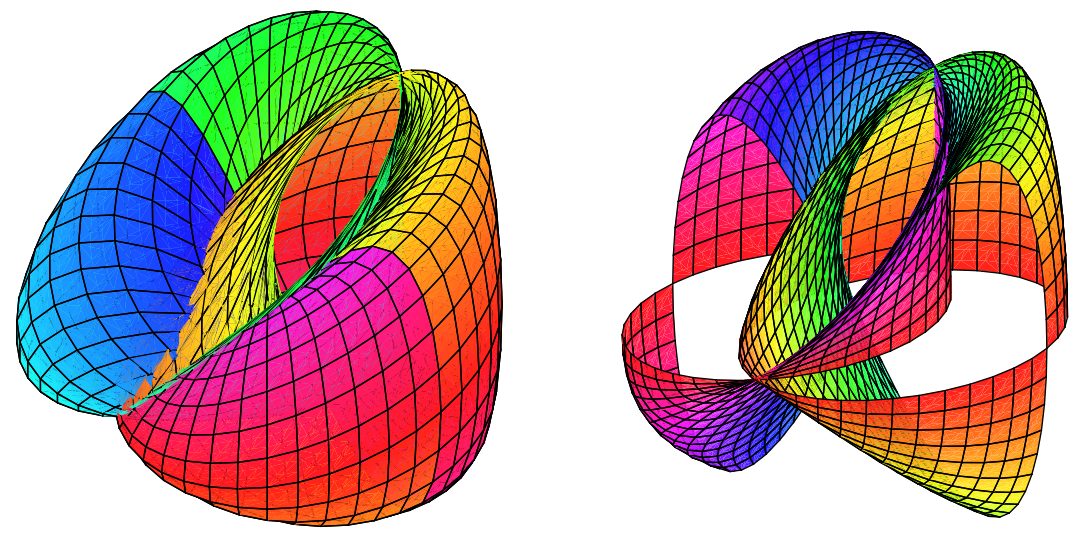

Figure 2. A section of an orbit for $n=3$. Right: cut-away view of same section. 


\section{Fundamental groups, covering spaces and spin(n)}

In order to make this exposition self-contained and to fix notation we review some background material that will be familiar to many readers.

Review of the fundamental group and covering spaces. Let $X$ be a topological space and let $[0,1] \subset \mathbb{R}$ be the closed unit interval. A path in $X$ is a continuous map $f:[0,1] \rightarrow X$. Two paths $f$ and $g$ from $x_{1}$ to $x_{2}$ are said to be homotopic if one can be continuously deformed into the other. This is obviously an equivalence relation, and we denote the equivalence class of $f$ by $[f]$. Of special interest will be loops, or closed paths that start and end at a distinguished base point $x \in X$, and we can define a multiplication of loops by concatenation. That is, $f \cdot g$ means first go around $f$ and then go around $g$. This operation is associative and is well defined when taking equivalence classes: $[f] \cdot[g]=[f \cdot g]$. The constant loop $e_{x}:[0,1] \rightarrow X$ given by $e_{x}(t)=x$ serves as the identity element for this operation and the loop $f^{-1}$ is the loop $f$ traversed in the opposite direction. We can then define the first homotopy group or the fundamental group, denoted $\pi_{1}(X, x)$, as the group of (equivalence classes of) loops in $X$ that start and end at $x$, along with this multiplication. If $x_{1}$ and $x_{2}$ are connected by a path in $X$, then $\pi_{1}\left(X, x_{1}\right)$ and $\pi_{1}\left(X, x_{2}\right)$ are isomorphic. Homeomorphic topological spaces have isomorphic fundamental groups, but the converse need not be true.

We will also require the notion of a covering. Let $(X, x),(Y, y)$ be topological spaces with base points $x$ and $y$ respectively. A map $p:(Y, y) \rightarrow(X, x)$ is a covering map if

(i) $p(y)=x$;

(ii) $p$ is continuous and surjective;

(iii) for every $x_{0} \in X$ there is an open neighborhood $U_{x_{0}} \subset X$ so that $p^{-1}\left(U_{x_{0}}\right)$ is a disjoint union of open sets $\left\{V_{\alpha}\right\}$ and so that for each $\alpha$, the map $p$ restricted to $V_{\alpha}$ is a homeomorphism of $V_{\alpha}$ onto $U_{x_{0}}$.

We then say that $(Y, y)$ is a covering space of $(X, x)$ and refer the the covering space along with the covering map as a cover of $(X, x)$. We will also use the standard results, roughly stated, that the composition of covers is a cover, and that the cover of a product is the product of the respective covers.

Remark 5.1. A topological space with trivial fundamental group is called simply connected. A covering space that is simply connected is called a universal covering space. It is unique up to homeomorphism.

We will need the notion of lifting a path from a space to a covering space.

Let $p:(Y, y) \rightarrow(X, x)$ be a covering map. Let $f:[0,1] \rightarrow X$ be a path starting at $x$. A lifting of $f$ is a path $\tilde{f}:[0,1] \rightarrow Y$ such that $p \circ \tilde{f}=f$. For the cases we 
are considering, these lifts are unique up to homotopy. That is, let $f$ be a path in $X$ beginning at $x$, and let $\tilde{f}$ and $\tilde{g}$ be two lifts of $f$ both beginning at $y$. Then $\tilde{f}$ is homotopic to $\tilde{g}$. In particular, $\tilde{f}$ and $\tilde{g}$ must end at the same point in $Y$.

Let $p:(Y, y) \rightarrow(X, x)$ be a covering map. A homeomorphism $h: Y \rightarrow Y$ is called a deck transformation or covering transformation if $p \circ h=p$. Clearly the collection of all such deck transformations is a group with the operation being composition of maps.

We will use the following fact to determine $\pi_{1}(G . \delta, \delta)$.

Theorem 5.2. [Massey 1991, Corollary 7.5] If $(Y, y)$ is a universal covering space of $(X, x)$, the group of deck transformations of $(Y, y)$ is isomorphic to $\pi_{1}(X, x)$. If $p:(Y, y): \rightarrow(X, x)$ is a covering map, then the order of $\pi_{1}(X, x)$ is equal to the cardinality of the set $p^{-1}(x)$.

Now consider the map $p_{1}: G \rightarrow G . \delta$ given by $g \mapsto g . \delta$. Since $p_{1}^{-1}(\delta)=$ $\{\gamma \in G \mid \gamma . \delta=\delta\}=G_{\delta}$ is discrete, Theorem E4 of [Hall 2003] has the following consequence.

Proposition 5.3. Let $G=\mathrm{SO}(n) \times \mathrm{SO}(n)$ and let 1 denote the identity element in $G$. Let $V$ be the vector space of $n \times n$ real matrices and let $G$ act on $V$ by

$$
(g, h) . v:=g^{t} v h, \quad(g, h) \in G, \quad v \in V .
$$

If $\delta \in V$ is a diagonal matrix with $n$ distinct eigenvalues, and if $G . \delta$ is the $G$-orbit of $\delta$, then the map $p_{1}:(G, \mathbf{1}) \rightarrow(G . \delta, \delta)$ given by $g \mapsto g . \delta$ is a covering map.

Said another way, $G$ is a fiber bundle over the orbit $G . \delta$ with projection map $(g, h) \mapsto(g, h) . \delta$ and discrete fiber $G_{\delta}$.

$\operatorname{Spin}(n)$. We now provide a brief review of the construction of the Lie group $\operatorname{Spin}(n)$ and the covering map from $\operatorname{Spin}(n)$ to $\operatorname{SO}(n)$. This abridged description should be sufficient for our purposes, but for a more complete discussion, see [Bröcker and tom Dieck 1985]. The presentation below borrows extensively from the excellent exposition in [Simon 1996].

Consider the vector space $\mathbb{R}^{n}$ with standard basis $\left\{e_{1}, \ldots, e_{n}\right\}$. We form $C(n)$, the Clifford algebra on $\mathbb{R}^{n}$, by declaring that multiplication is associative, distributive over addition, and obeys the relations $e_{i} e_{j}+e_{j} e_{i}=-2 \delta_{i j}$. This is just a fancy way of saying that the basis elements anti-commute and $e_{i}^{2}=-1$. If $I=i_{1} i_{2} \ldots i_{k}$ is a multiindex with $1 \leq i_{1}<\cdots<i_{k} \leq n$ we set $e_{0}=1$, we set $e_{I}=e_{i_{1}} e_{i_{2}} \ldots e_{i_{k}}$ and we set $|I|=k$. Then $C(n)$ is an algebra with basis $\left\{e_{I}\right\}$ and it follows that the dimension of $C(n)$ is $2^{n}$. We also have the subalgebra of even elements

$$
C(n)_{\mathrm{even}}=\left\{A \in C(n) \mid A \text { is a linear combination of } e_{I} \text { with }|I| \text { even }\right\} .
$$


Examples. We have canonical isomorphisms:

- $C(0) \cong \mathbb{R}$

- $C(1) \cong \mathbb{C}$ via the map $e_{1} \mapsto i=\sqrt{-1}$;

- $C(2) \cong \mathbb{H}$ (the quaternion algebra) via the map $e_{1} \mapsto i, e_{2} \mapsto j$ and so $e_{1} e_{2} \mapsto k$. Here, $i, j$, and $k$ are those elements in $\mathbb{W}$ with $i^{2}=j^{2}=k^{2}=-1$ and $i j=k$;

- we also have $C(3)_{\mathrm{even}} \cong \mathbb{U}$ via the map $e_{1} e_{2} \mapsto i, e_{1} e_{3} \mapsto j$, so

$$
\left(e_{1} e_{2}\right)\left(e_{1} e_{3}\right)=e_{2} e_{3} \mapsto k .
$$

We can define $\operatorname{Spin}(n)$ to be the invertible elements $S$ of $C(n)_{\text {even }}$ that (among other things) leave the vector space $W=\mathbb{R}^{n}$ invariant under conjugation:

$$
S W S^{-1} \subseteq W .
$$

Now consider the quadratic elements

$$
q_{i j}=\frac{1}{2} e_{i} e_{j},
$$

for $1 \leq i<j \leq n$, and observe that they obey the same commutation relations as the generators $L_{i j}$ of the Lie algebra $\mathfrak{s o}(n)$. Therefore these quadratic elements form a Lie algebra isomorphic to $\mathfrak{s o}(n)$, and so to get the group $\operatorname{Spin}(n)$ we exponentiate these quadratic elements:

$$
\begin{aligned}
S_{i j}(t):=\exp \left(t q_{i j}\right) & =1+\left(t q_{i j}\right)+\frac{1}{2 !}\left(t q_{i j}\right)^{2}+\frac{1}{3 !}\left(t q_{i j}\right)^{3}+\cdots \\
& =\cos (t / 2)+\sin (t / 2)\left(2 q_{i j}\right),
\end{aligned}
$$

since $q_{i j}^{2}=-1$. As $t$ goes from 0 to $4 \pi, S_{i j}(t)$ gives a copy of $U(1)$ in $\operatorname{Spin}(n)$ which is homeomorphic to a circle in the plane spanned by 1 and $2 q_{i j}$.

Now the elements $A$ in $\operatorname{Spin}(n)$ act on $\mathbb{R}^{n}$ by conjugation and this gives a representation of $\operatorname{Spin}(n)$ on $\mathbb{R}^{n}$. Consequently, we have a map

$$
R: \operatorname{Spin}(n) \rightarrow \operatorname{SO}(n, \mathbb{R})
$$

defined by

$$
A e_{i} A^{-1}=\sum_{i=1}^{n} R_{j i}(A) e_{j} .
$$

We now determine the matrix representation of the group elements

$$
S_{i j}(t):=\exp \left(t q_{i j}\right)=\cos (t / 2)+\sin (t / 2)\left(e_{i} e_{j}\right)
$$

by determining the action on the basis vectors. First observe that $e_{i} e_{j}$ commutes with $e_{k}$ when $k$ is equal to neither $i$ nor $j$, so in this case

$$
S_{i j}(t) e_{k} S_{i j}^{-1}(t)=\left(\cos (t / 2)+\sin (t / 2)\left(e_{i} e_{j}\right)\right) e_{k}\left(\cos (t / 2)-\sin (t / 2)\left(e_{i} e_{j}\right)\right)=e_{k} .
$$


Now conjugating $e_{i}$ by $S_{i j}(t)$ we have

$$
\begin{aligned}
S_{i j}(t) e_{i} S_{i j}^{-1}(t) & =\left(\cos (t / 2)+\sin (t / 2)\left(e_{i} e_{j}\right)\right) e_{i}\left(\cos (t / 2)-\sin (t / 2)\left(e_{i} e_{j}\right)\right) \\
& =\left(\cos (t / 2)+\sin (t / 2)\left(e_{i} e_{j}\right)\right]^{2} e_{i} \\
& =\left(\cos ^{2}(t / 2)-\sin ^{2}(t / 2)\right) e_{i}-2 \cos (t / 2) \sin (t / 2) e_{j} \\
& =\cos (t) e_{i}-\sin (t) e_{j} .
\end{aligned}
$$

A similar computation applied to $e_{j}$ gives

$$
S_{i j}(t) e_{j} S_{i j}^{-1}(t)=\sin (t) e_{i}+\cos (t) e_{j} .
$$

Therefore, conjugation by $S_{i j}(t)=\exp \left(t q_{i j}\right)$ induces a rotation by an angle $t$ in the $e_{i}, e_{j}$ plane. Since these rotations generate $\mathrm{SO}(n)$, this map is surjective.

The following result is well known (see [Simon 1996, Sections VII.6-VII.7] or [Bröcker and tom Dieck 1985, Section 1.6].

Proposition 5.4. $\operatorname{Spin}(n)$ is simply connected. If $A \in \operatorname{Spin}(n)$ and if $R(A)$ is the $n \times n$ matrix with entries $R_{j i}(A)$ described in (5-1) above, then the map $R:(\operatorname{Spin}(n), 1) \rightarrow(\mathrm{SO}(n, \mathbb{R}), 1)$ is a twofold universal covering map and a homomorphism of Lie groups. The symbol 1 denotes the unit elements in the respective groups.

\section{The fundamental group of a generic orbit}

We are now ready to determine the fundamental group for a generic orbit of maximum dimension. We will proceed by elaborating on some previously introduced ideas and connecting them together in order to invoke Theorem 5.2.

As before, $\delta \in D$ denotes an element in the cross-section with $n$ distinct eigenvalues. By Proposition 3.1, a typical element in its stabilizer $G_{\delta}$ can be represented by a diagonal matrix with each entry equal to \pm 1 , and where an even number of entries are equal to -1 . From now on, let $I=i_{1} i_{2} \cdots i_{k}$ be a multiindex with $1 \leq i_{1}<\cdots<i_{k} \leq n, k$ even and set $l=k / 2$. Let $S T_{I}$ be the element in $G_{\delta}$ with those entries that are equal to -1 indexed by $I$. For example, if $n=6$,

$$
S T_{1,2,3,5}=\left(\begin{array}{cccccc}
-1 & 0 & 0 & 0 & 0 & 0 \\
0 & -1 & 0 & 0 & 0 & 0 \\
0 & 0 & -1 & 0 & 0 & 0 \\
0 & 0 & 0 & 1 & 0 & 0 \\
0 & 0 & 0 & 0 & -1 & 0 \\
0 & 0 & 0 & 0 & 0 & 1
\end{array}\right)
$$

Using this notation, $G_{\delta}=\left\{\left(S T_{I}, S T_{I}\right):|I|\right.$ is even $\}$. 
Let $\tau=\left(t_{1}, \ldots, t_{l}\right)$ and let $\mathrm{SO}_{I}(\tau)$ be the matrix consisting of rotations by an angle $t_{j}$ in the planes indexed pairwise by $I$. These pairs are of the form $i_{2 m-1}, i_{2 m}$.

For example, if $I=1,2,3,5$ and $\tau=\left(t_{1}, t_{2}\right)$ then $\mathrm{SO}_{I}(\tau)$ rotates by an angle $t_{1}$ in the 1, 2 plane and by an angle $t_{2}$ in the 3,5 plane. For instance, if $n=6$,

$$
\mathrm{SO}_{1,2,3,5}(\tau)=\left(\begin{array}{cccccc}
\cos t_{1} & \sin t_{1} & 0 & 0 & 0 & 0 \\
-\sin t_{1} & \cos t_{1} & 0 & 0 & 0 & 0 \\
0 & 0 & \cos t_{2} & 0 & \sin t_{2} & 0 \\
0 & 0 & 0 & 1 & 0 & 0 \\
0 & 0 & -\sin t_{2} & 0 & \cos t_{2} & 0 \\
0 & 0 & 0 & 0 & 0 & 1
\end{array}\right) .
$$

Notice that $\mathrm{SO}_{1,2,3,5}(\tau)$ is equal to the matrix product $\mathrm{SO}_{1,2}\left(t_{1}\right) \mathrm{SO}_{3,5}\left(t_{2}\right)$. It should be easy to see that

Lemma 6.1. $S T_{I}=\mathrm{SO}_{I}( \pm \pi, \ldots, \pm \pi)$.

We next consider product of elements $S_{i j}(t) \in \operatorname{Spin}(n)$ and relate them to the corresponding elements in $\mathrm{SO}(n)$.

Lemma 6.2. Let $I=i_{1} i_{2} \cdots i_{k}$ be a multiindex with $k$ even and where

$$
i_{1}<i_{2}<\cdots<i_{k} .
$$

Set $l=k / 2$. Let $\tau=\left(t_{1}, \ldots, t_{l}\right)$ and let $\mathrm{SO}_{I}(\tau)$ be the matrix consisting of rotations by an angle $t_{j}$ in the planes indexed pairwise by $I$. Let $S_{i, j}(t)$ be defined as in $(5-2)$, and let $S_{I}(\tau)$ designate the product $S_{I}(\tau)=S_{i_{1} i_{2}}\left(t_{1}\right) S_{i_{3} i_{4}}\left(t_{2}\right) \cdots S_{i_{k-1} i_{k}}\left(t_{l}\right)$. Let $R: \operatorname{Spin}(n) \rightarrow \mathrm{SO}(n)$ be the covering map given by Proposition 5.4. Then $R\left(S_{I}(\tau)\right)=\mathrm{SO}_{I}(\tau)$.

Further, $e_{I}:=e_{i_{1}} e_{i_{2}} \cdots e_{i_{k}}$, we have $e_{I}=S_{I}(\pi, \ldots, \pi)$.

Proof. Since the entries in the multiindex $I$ are distinct, the designation $\mathrm{SO}_{I}(\tau)=$ $\mathrm{SO}_{i_{1} i_{2} \cdots i_{k}}(\tau)=\mathrm{SO}_{i_{1} i_{2}}\left(t_{1}\right) \mathrm{SO}_{i_{3} i_{4}}\left(t_{2}\right) \cdots \mathrm{SO}_{i_{k-1} i_{k}}\left(t_{l}\right)$ is unambiguous. Since the map $R$ is a representation, we have

$$
\begin{aligned}
R\left[S_{I}(\tau)\right] & =R\left[S_{i_{1} i_{2}}\left(t_{1}\right)\right] R\left[S_{i_{3} i_{4}}\left(t_{2}\right)\right] \cdots R\left[S_{i_{k-1} i_{k}}\left(t_{l}\right)\right] \\
& =\mathrm{SO}_{i_{1} i_{2}}\left(t_{1}\right) \mathrm{SO}_{i_{3} i_{4}}\left(t_{2}\right) \cdots \mathrm{SO}_{i_{k-1} i_{k}}\left(t_{l}\right)=\mathrm{SO}_{I}(\tau) .
\end{aligned}
$$

For the last assertion, note that (5-2) gives $e_{i} e_{j}=S_{i j}(\pi)$ for any $i, j$, since $\cos (\pi / 2)=0$ and $\sin (\pi / 2)=1$. Hence

$$
e_{I}=\left[e_{i_{1}} e_{i_{2}}\right]\left[e_{i_{3}} e_{i_{4}}\right] \cdots\left[e_{i_{k-1}} e_{i_{k}}\right]=S_{i_{1} i_{2}}(\pi) S_{i_{3} i_{4}}(\pi) \cdots S_{i_{k-1} i_{k}}(\pi)=S_{I}(\pi, \ldots, \pi),
$$

as required.

This next result is proven similarly. 
Lemma 6.3. Denote by $\pi^{+}$an l-tuple $\pi^{+}=( \pm \pi, \ldots, \pm \pi)$ with an even number of entries equal to $-\pi$ and denote by $\pi^{-}$an l-tuple $\pi^{-}=( \pm \pi, \ldots, \pm \pi)$ with an odd number of entries equal to $-\pi$. Let $S_{I}(\tau)$ and $e_{I}$ be as in the previous lemma. Then $S_{I}\left(\pi^{+}\right)=e_{I}$ and $S_{I}\left(\pi^{-}\right)=-e_{I}$.

Finally, let $\widetilde{\mathbf{1}}$ denote the unit element in $\widetilde{G}=\operatorname{Spin}(n) \times \operatorname{Spin}(n)$ and let $\mathbf{1}$ denote the unit element in $G=\operatorname{SO}(2, \mathbb{R}) \times \operatorname{SO}(2, \mathbb{R})$. Then $(\widetilde{G}, \widetilde{\mathbf{1}})$ is the universal covering space (in fact, a covering group) of $(G, 1)$ and the map

$$
\rho=R \times R:(\widetilde{G}, \widetilde{\mathbf{1}}) \rightarrow(G, \mathbf{1})
$$

is a fourfold covering map. Now recall the covering map $p_{1}:(G, \mathbf{1}) \rightarrow(G . \delta, \delta)$ from Proposition 5.3. It follows that the composition

$$
P=\rho \circ p_{1}:(\widetilde{G}, \widetilde{\mathbf{1}}) \rightarrow(G . \delta, \delta)
$$

is a covering map and that $\widetilde{G}$ is the universal covering space of the orbit $G . \delta$.

Definition 6.4. $E(n)=\left\{ \pm e_{I}:|I|\right.$ is even $\}$.

Observe that $E(n)$ is closed under multiplication since, if $e_{I} e_{J}=e_{K}$ then $|K|=$ $|I|+|J|$ when $I$ and $J$ are distinct indices, and the entries of $K$ contract in pairs when $I$ and $J$ have repeated entries. For example, $e_{1,2} e_{2,3}=-e_{1,3}$. Since $\left(e_{I}\right)^{-1}=$ $\pm e_{I}, E(n)$ is a group under multiplication. A computation very similar to that in Proposition 4.1 shows that $|E(n)|=2^{n}$.

Definition 6.5. Consider the set $\widetilde{E(n)}=\{(v, \pm v) \mid v \in E(n)\}$. This is a subgroup of $\widetilde{G}$ which is isomorphic to the group $E(n) \times \mathbb{Z}_{2}$ via the identifications $(v, 1) \mapsto(v, v)$ and $(v,-1) \mapsto(v,-v)$ for $v \in E(n)$.

Proposition 6.6. $P^{-1}(\delta)=\widetilde{E(n)}$.

Proof.

$$
\begin{aligned}
P\left[\left(e_{I}, e_{I}\right)\right] & =p_{1} \circ\left[R\left(e_{I}\right), R\left(e_{I}\right)\right], \\
\text { Lemma } 6.3 \Rightarrow & =p_{1} \circ\left[R \left(S_{I}\left(\pi^{+}\right), R\left(S_{I}\left(\pi^{+}\right)\right],\right.\right. \\
\text {Lemma } 6.2 \Rightarrow & =p_{1} \circ\left[\operatorname{SO}_{I}\left(\pi^{+}\right), \mathrm{SO}_{I}\left(\pi^{+}\right)\right], \\
\text {Lemma } 6.1 \Rightarrow & =p_{1} \circ\left[S T_{I}, S T_{I}\right], \\
& =\delta .
\end{aligned}
$$

The proofs of the other cases such as $P\left[\left(e_{I},-e_{I}\right)\right]=\delta$ are similar and hence $\widetilde{E(n)} \subseteq P^{-1}(\delta)$.

Now $p_{1}^{-1}(\delta)=\left\{\left(S T_{I}, S T_{I}\right):|I|\right.$ is even $\} \subseteq G$ has order $2^{n-1}$ (Proposition 4.1) and $\rho$ is a fourfold covering map $\widetilde{G} \rightarrow G$. Therefore the set $P^{-1}(\delta)$ has order $2^{n+1}$ which is equal to the order of $\widetilde{E(n)}$. 
The main result of this paper completely describes the fundamental group of a generic orbit.

Theorem 6.7. Let $G=\mathrm{SO}(n) \times \mathrm{SO}(n)$ and let $V$ be the vector space of $n \times n$ real matrices. Let $G$ act on $V$ via $(g, h) . v=g^{t} v h$. Let $\delta=\operatorname{diagonal}\left(d_{1}, d_{2}, \ldots, d_{n}\right) \in V$ with $d_{1}>d_{2}>\ldots>d_{n} \geq 0$, and let $G$. $\delta$ be the $G$-orbit of $\delta$ in $V$. Let $e_{1}, \ldots, e_{n}$ be the standard basis vectors in $\mathbb{R}^{n}$ and let $E(n)=\left\{ \pm e_{i_{1}} \ldots e_{i_{k}} \mid k\right.$ is even $\}$ be the group generated by the quadratic units $e_{i} e_{j}, i<j$ in the Clifford algebra on $\mathbb{R}^{n}$. Then the fundamental group $\pi_{1}(G . \delta, \delta)$ is isomorphic to $E(n) \times \mathbb{Z}_{2}$.

Proof. We will show that the group of deck transformations $\operatorname{Aut}(\widetilde{G}, P)$ on the universal covering $(\widetilde{G}, \mathbf{1})$ is isomorphic to $\widetilde{E(n)}$ which is isomorphic to $E(n) \times \mathbb{Z}_{2}$.

For each $\widetilde{\omega} \in \widetilde{E(n)}$ and $\widetilde{s} \in \widetilde{G}$ define the left translation map $\mathscr{L}_{\widetilde{\omega}}: \widetilde{G} \rightarrow \widetilde{G}$ by $\mathscr{L}_{\widetilde{\omega}}(\widetilde{S})=\widetilde{\omega} \widetilde{s}$, the operation on the right-hand side being multiplication in $\widetilde{G}$. It is a standard exercise that the set of all such translations $\mathbb{L}=\left\{\mathscr{L}_{\widetilde{\omega}} \mid \widetilde{\omega} \in \widetilde{E(n)}\right\}$ is a group that is isomorphic to $\widetilde{E(n)}$ via the map $\widetilde{\omega} \mapsto \mathscr{L}_{\widetilde{\omega}}$. Since $\widetilde{G}$ is a Lie group, each translation is continuous with a continuous inverse, hence a homeomorphism from $\widetilde{G}$ to $\widetilde{G}$. Furthermore, for each $\widetilde{v} \in \widetilde{E(n)}$, the composition $P \circ \mathscr{L}_{\widetilde{\omega}}(\widetilde{\mathcal{v}})=P(\widetilde{\omega} \widetilde{\mathcal{v}})=\delta$ so each $\mathscr{L}_{\widetilde{\omega}}$ is a deck transformation and therefore $\mathbb{L}$ is a subgroup of $\operatorname{Aut}(\widetilde{G}, P)$. $\operatorname{But} \operatorname{Aut}(\widetilde{G}, P)$ has order $2^{n+1}$ by Theorem 5.2, and since both these groups have the same order, they must be equal. By Theorem 5.2 again we have $\pi_{1}(G . \delta, \delta) \cong$ $\operatorname{Aut}(\widetilde{G}, P)=\mathbb{L} \cong \widetilde{E(n)} \cong E(n) \times \mathbb{Z}_{2}$.

\section{An illustration}

We conclude with an example for $n=6$ that further illustrates the previous constructions. The element

$$
S_{3,5}(t)=\exp \left[(t / 2) e_{3} e_{5}\right]=\cos (t / 2)+\sin (t / 2) e_{3} e_{5}
$$

in Spin(6) defined in (5-2) is homeomorphic to a circle lying in the plane spanned by 1 and $e_{3} e_{5}$ in the Clifford algebra $C(6)$, and which projects onto the rotation $\mathrm{SO}_{3,5}(t)$ in $\mathrm{SO}(6)$ via the representation $R$. Consider the path $\widetilde{f}:[0,4 \pi] \rightarrow \widetilde{G}$ given by $t \mapsto\left(S_{35}(t), S_{35}(t)\right)$.

Since $\widetilde{f}$ is homeomorphic to a circle and $\widetilde{G}$ is a simply connected covering group, $[\widetilde{f}]$ is trivial in $\pi_{1}(\widetilde{G}, \mathbf{1})$. Now as $t$ goes from 0 to $\pi$, we get a path $\widetilde{f}_{[0, \pi]}$ from $(1,1)$ to $\left(e_{3} e_{5}, e_{3} e_{5}\right)$ in $\widetilde{G}$ that projects down via $P$ to a loop $f:[0, \pi] \rightarrow G . \delta$ given by $f(t)=\left(\mathrm{SO}_{3,5}(t), \mathrm{SO}_{3,5}(t)\right) . \delta$. By uniqueness of path lifting, $f$ cannot be homotopic to the trivial loop since $\widetilde{f}_{[0, \pi]}$ is not trivial in $\widetilde{G}$. Similarly, as $t$ goes from $\pi$ to $2 \pi$, we get a path $\widetilde{f}_{[\pi, 2 \pi]}$ from $\left(e_{3} e_{5}, e_{3} e_{5}\right)$ to $(-1,-1)$ in $\widetilde{G}$ that also projects down to the loop $f$ in the orbit $G . \delta$. Not until $t$ travels the entire distance $[0,4 \pi]$ do we obtain the product $f^{4}$ in $G . \delta$ that lifts to the (trivial) loop $\widetilde{f}$ in $\widetilde{G}$. 
Thus, $[f]^{4}$ is trivial in $\pi_{1}(G . \delta, \delta)$. We chart here the information as the path $\tilde{f}$ is projected onto $G$ and then $G . \delta$ for the successive landmark values of $t$.

\begin{tabular}{c|ccc}
$t$ & $\widetilde{f}(t))$ & $\rho\left(\left(S_{3,5}(t), S_{3,5}(t)\right)\right)$ & $P\left(S_{3,5}(t), S_{3,5}(t)\right)$ \\
\hline 0 & $(1,1)$ & $\left(\Phi_{6}, \Phi_{6}\right)$ & $\delta$ \\
$\pi$ & $\left(e_{3} e_{5}, e_{3} e_{5}\right)$ & $\left(S T_{3,5}, S T_{3,5}\right)$ & $\delta$ \\
$2 \pi$ & $(-1,-1)$ & $\left(\Phi_{6}, \Phi_{6}\right)$ & $\delta$ \\
$3 \pi$ & $\left(-e_{3} e_{5},-e_{3} e_{5}\right)$ & $\left(S T_{3,5}, S T_{3,5}\right)$ & $\delta$ \\
$4 \pi$ & $(1,1)$ & $\left(\Phi_{n}, \Phi_{n}\right)$ & $\delta$
\end{tabular}

As in the previous discussion regarding deck transformations in the proof of Theorem 6.7, we can translate the loop $\widetilde{f}$ via left multiplication by the element $\left(e_{1} e_{2}, e_{1} e_{2}\right) \in \widetilde{E(n)}$. This gives us the loop $\widetilde{g}:[0,4 \pi] \rightarrow \widetilde{G}$ given by $t \mapsto(v(t), v(t))$ where

$$
v(t)=e_{1} e_{2}\left[\cos (t / 2)+\sin ((t / 2)) e_{3} e_{5}\right]=\cos (t / 2) e_{1} e_{2}+\sin (t / 2) e_{1} e_{2} e_{3} e_{5} .
$$

This is a loop starting at $e_{1} e_{2}$ which lies in the plane spanned by $e_{1} e_{2}$ and $e_{1} e_{2} e_{3} e_{5}$ in the Clifford algebra $C(6)$.

We check that

$$
v^{-1}(t)=\left[-\cos (t / 2) e_{1} e_{2}+\sin (t / 2) e_{1} e_{2} e_{3} e_{5}\right]
$$

and that conjugating the basis vectors $e_{i} \in \mathbb{R}^{6}$ by $v(t)$ produces the map $R$ which takes $v(t)$ to the rotation

$$
R(v(t))=\left(\begin{array}{cccccc}
-1 & 0 & 0 & 0 & 0 & 0 \\
0 & -1 & 0 & 0 & 0 & 0 \\
0 & 0 & \cos t & 0 & \sin t & 0 \\
0 & 0 & 0 & 1 & 0 & 0 \\
0 & 0 & -\sin t & 0 & \cos t & 0 \\
0 & 0 & 0 & 0 & 0 & 1
\end{array}\right) \in \mathrm{SO}(6)
$$

As above, the projection $P$ maps $\widetilde{g}_{[0, \pi]}$ to the loop $g(t)=R(v(t)) . \delta$ in the orbit $G . \delta$ and $[g]^{4}$ is trivial. Here is part of this information for the path $\widetilde{g}$ :

\begin{tabular}{c|ccc}
$t$ & $\widetilde{g}(t)$ & $\rho(\widetilde{g}(t)))$ & $P(\widetilde{g}(t)))$ \\
\hline 0 & $\left(e_{1} e_{2}, e_{1} e_{2}\right)$ & $\left(S T_{1,2}, S T_{1,2}\right)$ & $\delta$ \\
$\pi$ & $\left(e_{1} e_{2} e_{3} e_{5}, e_{1} e_{2} e_{3} e_{5}\right)$ & $\left(S T_{1,2,3,5}, S T_{1,2,3,5}\right)$ & $\delta$ \\
$2 \pi$ & $\left(-e_{1} e_{2},-e_{1} e_{2}\right)$ & $\left(S T_{1,2}, S T_{1,2}\right)$ & $\delta$ \\
$3 \pi$ & $\left(-e_{1} e_{2} e_{3} e_{5},-e_{1} e_{2} e_{3} e_{5}\right)$ & $\left(S T_{1,2,3,5}, S T_{1,2,3,5}\right)$ & $\delta$
\end{tabular}

By considering the loops in the orbit $G . \delta$ that lift to the path from

$$
(1,1) \rightarrow\left(e_{1} e_{2}, e_{1} e_{2}\right) \rightarrow\left(e_{1} e_{2} e_{3} e_{5}, e_{1} e_{2} e_{3} e_{5}\right)
$$


in $\widetilde{G}$ we see that $g$ and $f$ cannot be homotopic, so $[g]$ and $[f]$ are distinct elements in $\pi_{1}(G . \delta, \delta)$.

\section{Final remarks on the general case}

Determining the first homotopy group for the orbits in the more general case, when the representative element $d$ in the cross-section contains eigenvalues with multiplicities greater than 1 , does not lend itself to such direct construction since the map $G \rightarrow G . d$ is not a covering map.

\section{Acknowledgments}

We would like to thank Professors C. Benson, G. Ratcliff and A. Smith for many helpful conversations.

\section{References}

[Bröcker and tom Dieck 1985] T. Bröcker and T. tom Dieck, Representations of compact Lie groups, Grad. Texts in Math. 98, Springer, New York, 1985. MR 86i:22023 Zbl 0581.22009

[Hall 2003] B. C. Hall, Lie groups, Lie algebras, and representations, Grad. Texts in Math. 222, Springer, New York, 2003. MR 2004i:22001 Zbl 1026.22001

[Massey 1991] W. S. Massey, A basic course in algebraic topology, Grad. Texts in Math. 127, Springer, New York, 1991. MR 92c:55001 Zbl 0725.55001

[Simon 1996] B. Simon, Representations of finite and compact groups, Grad. Studies in Math. 10, American Mathematical Society, Providence, RI, 1996. MR 97c:22001 Zbl 0840.22001

Received: 2008-04-08 Revised: Accepted: 2009-09-28

czarn005@rangers.uwp.edu Department of Mathematics, University of Wisconsin-Parkside, 900 Wood Rd., P.O. Box 2000, Kenosha, WI 53141-2000, United States

hower@uwec.edu Department of Mathematics, University of Wisconsin-Eau Claire, 508 Hibbard Humanities Hall, Eau Claire, WI 54702-4004, United States http://www.uwec.edu/math/Faculty/howe.htm

Aaron.D.McTavish@uwsp.edu

Department of Mathematical Sciences,

University of Wisconsin-Stevens Point, Stevens Point, WI 54481-3897, United States 